\title{
Self-Organized Growth of Alloy Superlattices
}

P. Venezuela*, J. Tersoff ${ }^{\dagger}$, J. A. Floro ${ }^{\ddagger}$, E. Chason ${ }^{\ddagger}$,

D. M. Follstaedt $\ddagger^{\ddagger}$, Feng Liu* \& M. G. Lagally*

- University of Wisconsin, Madison, WI 53706

${ }^{\dagger} I B M$ Research Division, T. J. Watson Research Center,

P. O. Box 218, Yorktown Heights, NY 10598

$\ddagger$ Sandia National Laboratories, Albuquerque, NM 87185-1415

$\S$ Present Address: Brown University, Division of Engineering, Providence, RI 02912. 


\section{DISCLAIMER}

This report was prepared as an account of work sponsored by an agency of the United States Government. Neither the United States Government nor any agency thereof, nor any of their employees, make any warranty, express or implied, or assumes any legal liability or responsibility for the accuracy, completeness, or usefulness of any information, apparatus, product, or process disclosed, or represents that its use would not infringe privately owned rights. Reference herein to any specific commercial product, process, or service by trade name, trademark, manufacturer, or otherwise does not necessarily constitute or imply its endorsement, recommendation, or favoring by the United States Government or any agency thereof. The views and opinions of authors expressed herein do not necessarily state or reflect those of the United States Government or any agency thereof. 


\section{DISCLAIMER}

Portions of this document may be illegible in electronic image products. Images are produced from the best available original document. 
We predict theoretically and demonstrate experimentally the spontaneous formation of a superlattice during crystal growth. When a strained alloy grows by "step flow", the steps at the surface form periodic bunches. The resulting modulated strain biases the incorporation of the respective alloy components at different steps in the bunch, leading to the formation of a superlattice. X-ray diffraction and electron microscopy for SiGe grown on Si give clear evidence for such spontaneous superlattice formation.

Self-organization is ubiquitous in nature. Yet only recently have attempts been made to fabricate useful nanoscale structures by controlled self-organization. Fascinating nanoscale materials have been formed by condensing particles from a suspension [1]; and potential device structures such as "quantum dots" have been made via self-organization on a surface [2] or in stacked layers grown sequentially [3].

Here we report self-organization of a nanostructured electronic material in a continuous growth process. Specifically, we theoretically predict and experimentally observe selfassembly of a superlattice (i.e. a periodically layered structure) during continuous growth of a strained alloy. Such superlattices have wide application in optoelectronics, and potential applications as novel thermoelectrics [4].

Superlattices are generally grown by alternate deposition of two materials. The possibility of "natural" superlattices has been suggested previously, based on layered structures seen in epitaxial growth of alloys that are unstable against spinodal decomposition [5]. However, only one instance of well-organized growth has been reported in such systems [6], and theoretical explanations remain speculative $[7,8]$.

Here we address self-organization induced by strain rather than by spinodal decomposition. Strain arises when the deposited material accommodates itself to the (slightly different) lattice constant of the substrate. We propose a detailed theory based on the wellestablished understanding of epitaxial growth. For "step-flow growth", the theory predicts that spontaneous superlattice formation is a quite general phenomenon.

Our conclusions are supported by our experimental observation of spontaneous superlat- 
tice formation in $\mathrm{SiGe}$, a system which has no tendency toward spinodal decomposition. If such growth can be controlled and optimized, it will provide a simple and efficient way of producing nanostructured materials with novel electronic and optical properties. Such materials could have a variety of applications, even if they are far less perfect than superlattices grown by the traditional painstaking approach.

Some periodic modulation of alloy composition is occasionally seen in semiconductor growth, due to artifacts of the experimental procedure. These may include rotation of the wafer under a nonuniform flux of material, or periodic variations in growth temperature as the heater cycles on and off. Thus truly self-organized modulations could be easily overlooked. Here we are able to rule out all known experimental artifacts, providing strong evidence for the theoretical interpretation. The generality of the effect suggests that such composition variations may also be a common unintended occurrence in growth of alloy layers, with the potential to degrade device performance.

We sketch the main aspects of the theory before describing the theoretical and experimental results. Semiconductor devices are typically grown on a "vicinal" surface, a staircase of atomically flat terraces separated by atomic-height steps. Atoms are deposited on the surface (by evaporation or vapor decomposition), and they diffuse as "adatoms" on the terraces. Step motion arises from attachment or detachment of these adatoms at the step.

The adatom density on a terrace obeys the diffusion equation, subject to boundary conditions of equilibrium at the steps. (We do not include any step-edge diffusion barriers.) Solving this equation we find the current of adatoms. The discontinuity in this current at a step gives the net attachment or detachment rate, and hence the step velocity. The physics of strain and alloying enters solely through the composition-dependent chemical potential at the steps, which determines the local adatom density and hence the adatom diffusion, step motion, and crystal growth [9-11].

Recently Liu et al. [12] showed that such growth leads to periodic patterns of step bunches on the crystal surface. Here we consider growth of an alloy, in which the elements differ in their size and surface mobility. The step bunches affect the surface elastic field, giving 
different strains at different steps. The smaller adatoms will be incorporated preferentially at steps having relatively compressive strain, and larger adatoms at relatively tensile steps $[10,13]$. In addition, steps having lowest absolute strain are favored overall, and atoms of the more mobile species can more readily reach these preferred steps [11].

The result is an ordered pattern of composition modulation, as shown in Fig. 1. We believe that this is the first actual calculation showing spontaneous superlattice formation in any system. Note the remarkable regularity of the structure, which appears to be independent of initial conditions and robust against noise.

The equations describing alloy decomposition are nonlinear, and so inherently difficult to solve. We therefore consider the limit of only a small difference in size and/or mobility between the alloy elements, so that we can linearize the problem. In this case, and making use of prior results $[10,11]$, we can write the evolution of surface morphology as

$$
\dot{u}_{m}=\frac{1}{2}\left(u_{m+1}-u_{m-1}\right)+\bar{\lambda}^{3} \Phi_{m} .
$$

Here $u_{m}=\left(x_{m} / L_{a v}\right)-m-F t$ is the step displacement, written for convenience in dimensionless units, relative to an ideal train of equispaced steps; $x_{m}$ is the actual step position; $L_{a v}$ is the average step separation (fixed by the surface "vicinal angle"), and $F$ the total incident flux (the growth rate) in monolayers per unit time. The corresponding dimensionless step velocity is $\dot{u}_{m}=d u_{m} / d \tau$, where $\tau=F t$ is the dimensionless time. For an alloy of two components, indexed by the subscript $\nu, \lambda_{\nu}=\left[\exp \left(\bar{\mu}_{\nu}-E_{\nu} / k T\right) D_{\nu} M \bar{\varepsilon}^{2} \beta h / F k T\right]^{1 / 3} / L_{a v}$ characterizes the strain-driven diffusion for component $\nu$. Here $\bar{\mu}_{\nu}$ is the average chemical potential, $E_{\nu}$ and $D_{\nu}$ are the energy and mobility of an adatom of component $\nu, T$ is the temperature, $M$ is the product of the atomic volume and an elastic constant, $\bar{\varepsilon}$ is the misfit strain, $\beta$ is a ratio between elastic constants, and $h$ is the step height. Only the average $\bar{\lambda}=\left(\lambda_{\nu}+\lambda_{-\nu}\right) / 2$ enters Eq. (1), where the subscript $-\nu$ denotes the component other than v. Finally,

$$
\Phi_{m}=\frac{S_{m+1}-S_{m}}{u_{m+1}-u_{m}+1}-\frac{S_{m}-S_{m-1}}{u_{m}-u_{m-1}+1},
$$


where $S_{m}=\sum_{n \neq m}\left(u_{m}+m-u_{n}-n\right)^{-1}-l_{0}^{2} \sum_{n \neq m}\left(u_{m}+m-u_{n}-n\right)^{-3}$, and $l_{0}$ is the minimum-energy distance between two isolated steps, in units of $L_{a v}$.

The dynamical evolution of the surface morphology is determined by numerical integration of the step velocity, Eq. (1). The behavior is controlled by two parameters, $l_{0}$ and $\bar{\lambda}$. These reflect respectively the short-range strain around the step (due to details of atomic structure) and the long-range strain field (because the step changes the elastic boundary condition for the misfit strain).

The alloy decomposition at the steps is given by

$$
\overrightarrow{\Delta c_{\nu}}=\bar{c}_{\nu} \bar{c}_{-\nu} \alpha_{\nu} \vec{\Psi}\left[\mathbf{I}-\gamma \bar{\lambda}^{3} \mathbf{A}\right]^{-1}
$$

Here $\overrightarrow{\Delta c_{\nu}}$ gives the deviation from the average composition $\bar{c}_{\nu}$. Its $m$ th component refers to step $m$. The $m$ th component of $\vec{\Psi}$ is $\bar{\lambda}^{3} \Phi_{m} /\left(1+\dot{u}_{m}\right)$; I is the identity matrix; and $\mathbf{A}$ is a cyclic tridiagonal matrix with $A_{m, m \pm 1}= \pm\left(1+\dot{u}_{m}\right)^{-1}\left(u_{m \pm 1}-u_{m} \pm 1\right)^{-1}$ and $A_{m, m}=$ $-\left(A_{m, m+1}+A_{m, m-1}\right)$. A detailed derivation of these results will be given elsewhere.

Given the surface morphology, there are two important (and competing) terms which determine the alloy decomposition. The difference in size and mobility of the two species are reflected in

$$
\alpha_{\nu}=\Delta \varepsilon_{\nu} / \bar{\varepsilon}+3 \Delta \lambda_{\nu} / \bar{\lambda}
$$

where $\Delta \varepsilon_{\nu} / \bar{\varepsilon}$ is the fractional misfit difference, with $\Delta \varepsilon_{\nu}=\left(a_{-\nu}-a_{\nu}\right) / a_{0}, a_{\nu}$ and $a_{0}$ being the lattice parameters of component $\nu$ and of the substrate, respectively; and $\Delta \lambda_{\nu} / \bar{\lambda}$ is the fractional difference in mobility, relative to the average, where $\Delta \lambda_{\nu}=\lambda_{\nu}-\lambda_{-\nu}$. For small differences, the degree of decomposition is directly proportional to $\alpha$. Note that the effects of atomic size and mobility difference can add or partially cancel, depending on the relative signs [11].

The other important parameter is

$$
\gamma=\bar{c}_{\nu} \bar{c}_{-\nu} \frac{L_{a v}}{\beta h} \frac{g_{\nu}^{\prime \prime}}{M \bar{\varepsilon}^{2}}
$$


This acts in the opposite way from $\alpha_{\nu}$, to suppress decomposition. Here $g_{\nu}^{\prime \prime}$ is the second derivative, with respect to composition, of the free energy of mixing of the alloy. As long as the alloy is stable against spinodal decomposition, $g_{\nu}^{\prime \prime}>0$ and so $\gamma>0$. The larger $\gamma$ is, the smaller the decomposition $\overrightarrow{\Delta c}_{\nu}$. Other factors being equal, the alloy decomposition will be largest in systems where the critical temperature $T_{c}$ for spinodal decomposition is not far below the growth temperature $T$, since $\gamma \rightarrow 0$ as $T \rightarrow T_{c}$.

An example of the calculated growth is shown in Fig. 1 , for $l_{0}=0.192$ and $\bar{\lambda}=0.446$. Increasing the growth rate reduces $\bar{\lambda}$, giving smaller bunches [12] and hence a shorter superlattice period. The simulation included 120 steps, with periodic boundary conditions to eliminate end effects. The vertical axis in Fig. 1 has been exaggerated for clarity. The violet and red regions are enriched in one or the other component, while the yellow regions have composition close to the average value.

Because of the combination of short-range and long-range strains around each step, the total strain (and hence the chemical potential) is higher at the top and bottom of a stepbunch, and lower in the middle. The more mobile atoms are more successful at reaching the favored mid-bunch sites, and so are preferentially incorporated there. The atomic size similarly biases the incorporation of the two types of atoms - e.g. for compressive stress, the strain is relatively less compressive in mid-bunch, favoring incorporation of the larger atoms there.

Note that in Fig. 1 the superlattice is misoriented in relation to the crystallographic plane (horizontal direction) by an angle which is comparable to the surface vicinal angle, but in the opposite direction. This is due to the complex step dynamics, in which free steps are continually ejected from the base of some bunch and then captured by the adjacent bunch [12].

We have repeated the simulation for several values of $\gamma$. In general the pattern of the decomposition reflects the surface morphology, and so is unaffected by $\gamma$. However, $\gamma$ has a strong effect on the magnitude of the decomposition. Near the critical temperature $T_{c}$ for spinodal decomposition, $\gamma$ is small and the decomposition is quite large. However, it is 
difficult to evaluate $\gamma$ for specific real systems, because $T_{c}$ is not accurately known.

In Fig. 2 we show a cross-sectional Transmission Electron Microscope (TEM) image for a $\mathrm{Si}_{0.84} \mathrm{Ge}_{0.16}$ alloy grown by $\mathrm{MBE}$ on $\mathrm{Si}(001)$ at $380^{\circ} \mathrm{C}$, along with $\mathrm{X}$-ray diffraction data. The superlattice modulation is clearly visible, despite the "noisiness" of the image. (Viewing the image obliquely from the side enhances the clarity.) The X-ray diffraction provides a more quantitative measure. Each peak in the diffracted intensity indicates the presence of some composition modulation with the period indicated. Comparison with calculated diffraction intensities indicate that the composition modulation between layers is on the order of $10 \%$. We conclude that the sample has multiple domains, and that each peak originates in a distinct region having that periodicity.

Note that the separation between the principal diffraction peaks in Fig. 2 corresponds to an integer number of atomic double-layers $(\sim 2.7 \mathrm{~A})$. This is consistent with the proposed mechanism. The average bunch size may vary across the sample due to small differences in local surface miscut, flux or temperature. But bunches can differ only by integer numbers of steps; and if bunches of different size coexist they tend to "phase separate" into separate regions of uniform bunch size [12]. Such regions will have periods differing by integer numbers of atomic layers. (SiGe is well known to show step "pairing", and the dominant spacing in Fig. 2 corresponds to the bilayer step height.) We know of no artifacts (such as wafer rotation) which could cause such a sequence of peaks, with spacings tied to the step height. This is strong evidence that the experiment reflects the same mechanism for spontaneous superlattice formation as in our simulations.

There is still much to be understood in this problem. We have not yet identified the precise experimental conditions for reproducibly growing superlattices. And the quantitative predictions of the theory will be affected if we include other effects such as diffusion barriers, step permeability, and other step-repulsion mechanisms. But none of these effects will eliminate the basic morphological self-organization, and resulting periodic modulation of the alloy composition. These appear to be very robust effects, as long as growth takes place in the step-flow mode described. 


\section{REFERENCES}

[1] Murray, C. B., Kagan, C. R. \& Bawendi, M. G. Self-organization of CdSe nanocrystallites into three-dimensional quantum dot superlattices. Science 270, 1335-1338 (1995).

[2] Petroff, P. M., Medeiros-Ribeiro, G. Three-dimensional carrier confinement in straininduced self-assembled quantum dots. MRS Bulletin 21 No. 4, 50-54 (1996).

[3] Tersoff, J., Teichert, C. \& Lagally, M. G. Self-organization in growth of quantum dot superlattices. Phys. Rev. Lett. 76, 1675-1678 (1996).

[4] Mahan, G. D. \& Woods L. M. Multilayer thermionic refrigeration. Phys. Rev. Lett. 80, 4016-4019 (1998).

[5] Norman, A. G., Seong, T.Y, Fergunson, I. T., Booker, G. R. \& Joyce, B. A. Structural studies of natural superlattices in group III-V alloy epitaxial layers. Semicond. Sci. Technol. 8, S9-S15 (1993).

[6] Ahrenkiel, S. P. et al. Self-organized formation of compositionally modulated $\mathrm{ZnSe}_{1-x} \mathrm{Te}_{x}$ superlattices. Phys. Rev. Lett. 75, 1586-1589 (1995).

[7] Barabási, A. Self-organized superlattices formation in II-IV and III-V semiconductors. Appl. Phys. Lett. 70, 764-766 (1997).

[8] Tersoff, J. Spinodal decomposition during step-flow growth. Phys. Rev. B 56, R4394R4397 (1997).

[9] Tersoff, J., Phang, Y. H., Zhenyu Zhang \& Lagally, M. G. Step-bunching instability of vicinal substrates under stress. Phys. Rev. Lett. 75, 2730-2733 (1995).

[10] Tersoff, J. Stress-driven alloy decomposition during step-flow growth. Phys. Rev. Lett. $77,2017-2020$ (1996).

[11] Venezuela, P. \& Tersoff, J. Alloy decomposition during growth due to mobility differences. Phys. Rev. B (in press). 
[12] Liu F., Tersoff J. \& Lagally, M. G. Self-organization of steps in growth of strained films on vicinal substrates. Phys. Rev. Lett. 80, 1268-1271 (1998).

[13] Guyer, J. E. \& Voorhees P. W. Morphological stability of alloy thin films. Phys. Rev. Lett. 74, 4031-4034 (1995).

Acknowledgments. P. V. was supported by the Brazilian agency FAPESP.

Correspondence and requests for materials should be addressed to J. T.

Sandia is a multiprogram laboratory operated by Sandia Corporation, a Lockheed Martin Company, for the United States Department of Energy under contract DE-AC04-94AL85000. 


\section{FIGURES}

FIG. 1. Cross-sectional view of decomposition for simulation with $\bar{\lambda}=0.446$ and $l_{0}=0.192$. The vertical axis has been exaggerated for clarity. The violet and red regions are enriched in one or the other component, while the yellow regions have composition very close to the average composition.

FIG. 2. Measurements for a $\mathrm{Si}_{0.84} \mathrm{Ge}_{0.16}$ alloy grown on $\mathrm{Si}(001)$. (a) TEM image. Image is 1450 $\AA \times 1450 \AA$, with the top edge toward the growth surface. Despite "noise", horizontal layers with spacings around $3 \mathrm{~nm}$ are clearly visible. (b) X-ray diffraction intensity (from $\theta-2 \theta \mathrm{scan}$ ), versus periodicity (from $X$-ray wavelength $\lambda$ and scattering angle $\theta$ ). The arrows indicate successive major peaks differing by the bilayer step height. 
Fig. 1 J. Tersoff

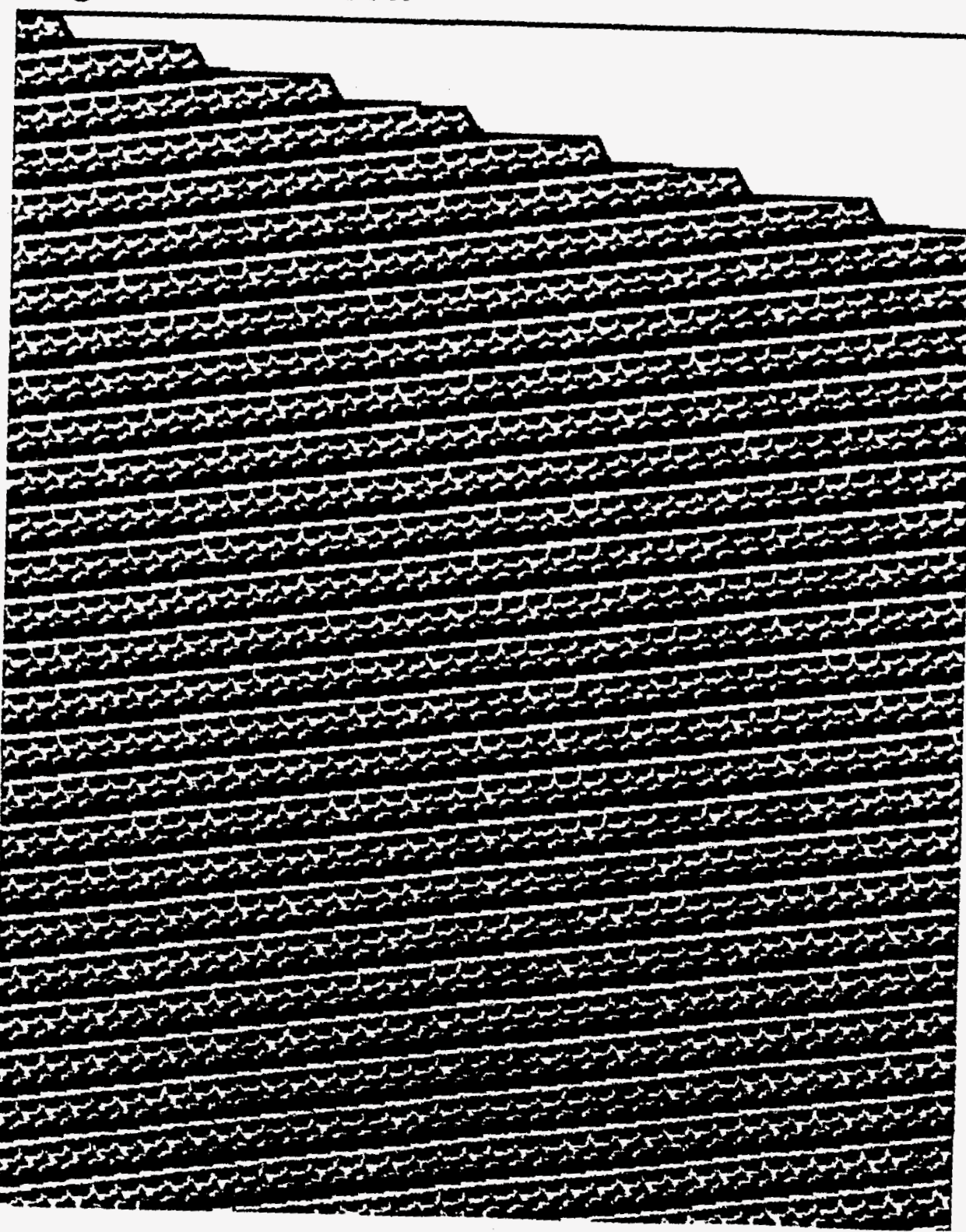




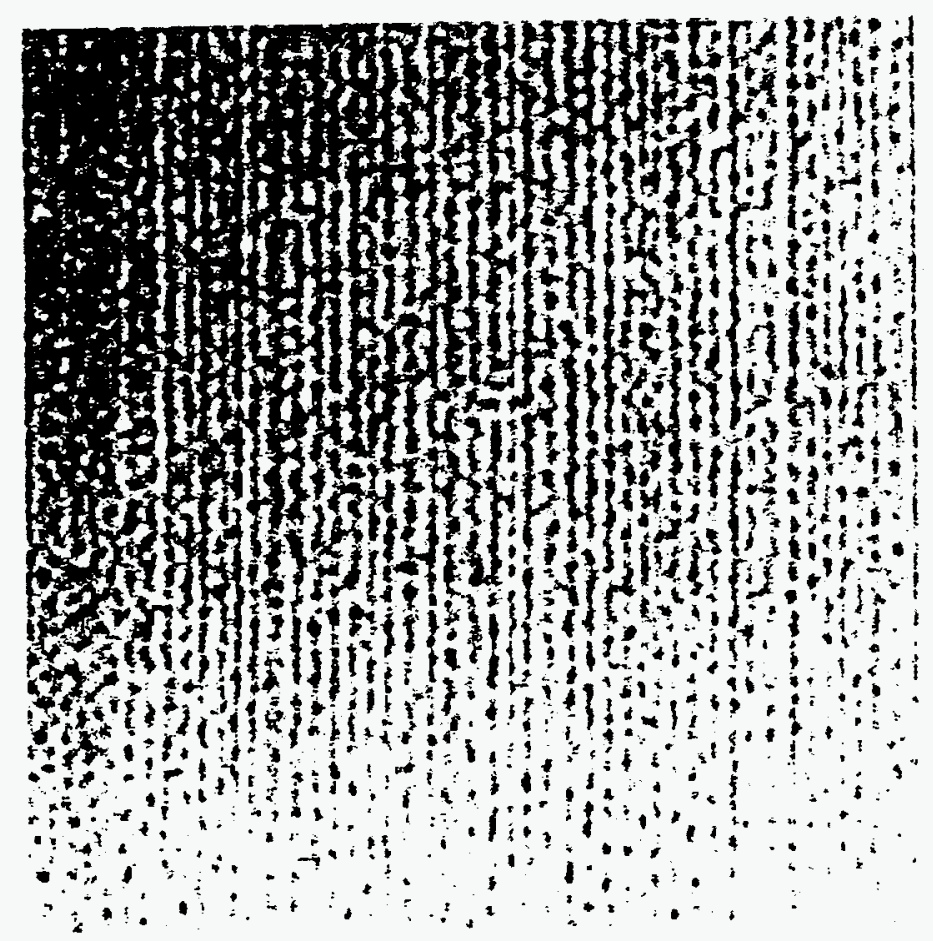


Fig. 2b

J. Tersoff

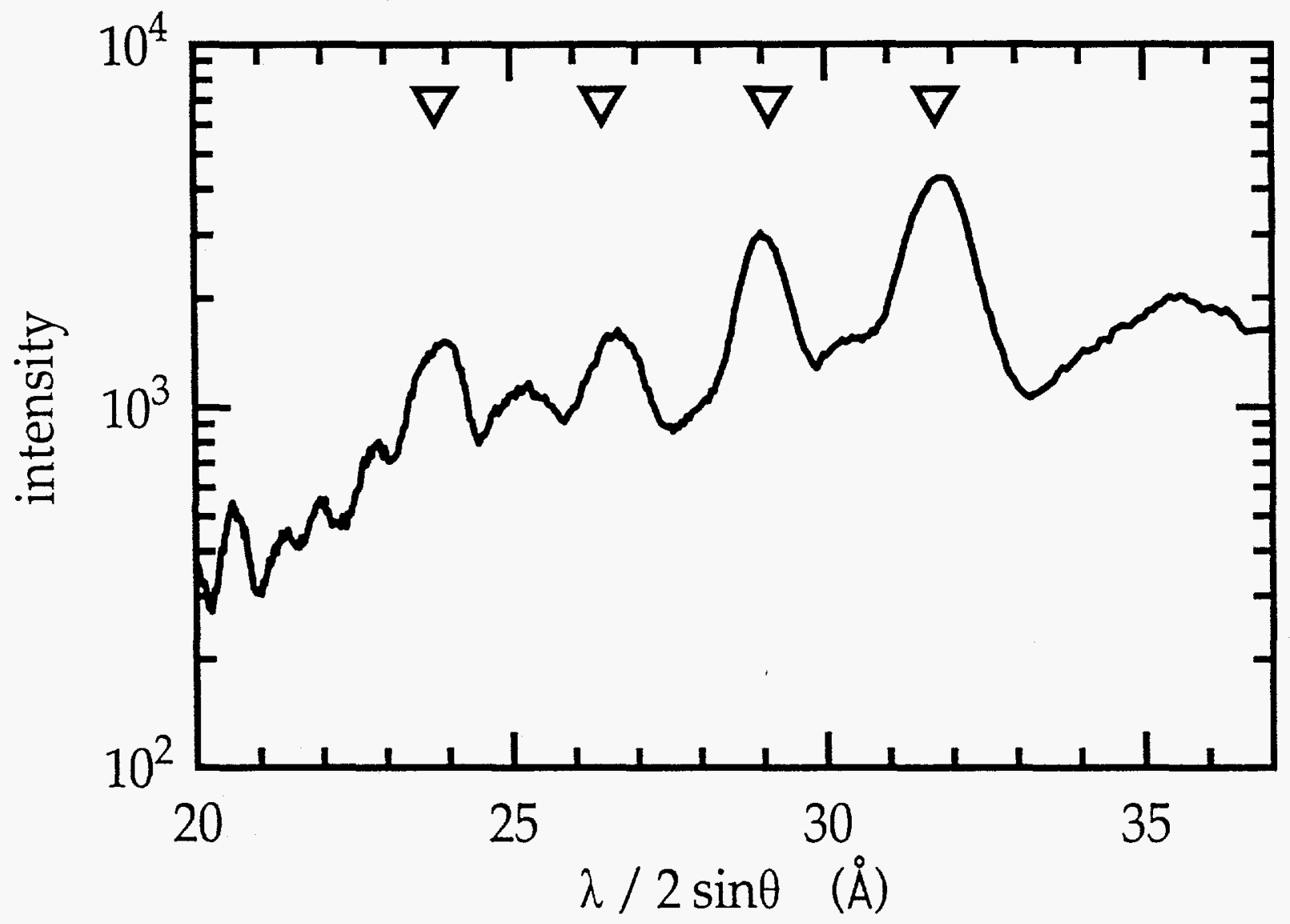

